\title{
Linx
}

Revue des linguistes de l'université Paris X Nanterre

$12 \mid 2002$

«Comme la lettre dit la vie »

\section{Narration et dialogue : un mais de transition en ancien français}

\section{Amalia Rodríguez Somolinos}

\section{(2) OpenEdition}

\section{Journals}

Édition électronique

URL : http://journals.openedition.org/linx/1311

DOI : 10.4000/linx.1311

ISSN : 2118-9692

\section{Éditeur}

Presses universitaires de Paris Nanterre

\section{Édition imprimée}

Date de publication : 1 octobre 2002

Pagination : 236-245

ISSN : 0246-8743

\section{Référence électronique}

Amalia Rodríguez Somolinos, «Narration et dialogue : un mais de transition en ancien français », Linx [En ligne], 12 | 2002, mis en ligne le 10 octobre 2012, consulté le 01 mai 2019. URL : http:// journals.openedition.org/linx/1311 ; DOI : 10.4000/linx.1311

Ce document a été généré automatiquement le 1 mai 2019.

Département de Sciences du langage, Université Paris Ouest 


\title{
Narration et dialogue : un mais de transition en ancien français
}

\author{
Amalia Rodríguez Somolinos
}

\section{Introduction}

1 Le fonctionnement sémantique de la conjonction mais, extrêmement complexe, avait échappé aux études traditionnelles, qui ne possédaient pas les outils théoriques permettant d'aborder sa description. Depuis une vingtaine d'années cependant, mais fait l'objet de nombreuses études. Ce sont essentiellement les travaux d'Oswald Ducrot et de Jean-Claude Anscombre qui ont ouvert la voie et qui ont permis de donner une description sémantique de mais. 0 . Ducrot a en effet construit au départ sa théorie de l'argumentation dans la langue en s'appuyant fortement sur la description sémantique de plusieurs connecteurs et opérateurs argumentatifs, dont surtout mais.

Dans un article très connu, Anscombre et Ducrot (1977) établissent l'existence en français de deux mais entièrement distincts: un mais argumentatif et un mais réfutatif, qui possèdent des propriétés syntaxiques et discursives différentes. Mais argumentatif est un connecteur concessif qui équivaut à l'espagnol pero et à l'allemand aber. Mais réfutatif équivaut à l'espagnol sino et à l'allemand sondern. Cette distinction établie, Oswald Ducrot approfondit par la suite l'analyse de mais argumentatif en français moderne. Il s'intéresse ainsi aux effets de sens que produit mais argumentatif dans différentes séquences textuelles. Il aborde dans Ducrot (1980) l'étude des emplois de mais dans des séquences narratives ou descriptives. Ducrot et al. (1980) réalise une classification des différents emplois de mais dans le dialogue, à partir de l'analyse d'une pièce de théâtre de Feydeau. Ces travaux, portant surtout sur des textes littéraires modernes, ont servi de point de départ à d'autres chercheurs abordant l'étude de mais argumentatif en français, notamment Adam (1990), Maingueneau (1990) et Rabatel (1999).

3 Si les travaux sur mais en français moderne sont maintenant assez nombreux, la description de mais en diachronie n'a pas suscité autant d'intérêt. Il faut surtout noter Kleiber (1978) qui présente, en s'appuyant sur les travaux de Ducrot, une description 
sémantique de mais et de ainz en ancien français. On retrouve dans cette langue les deux mais décrits par Ducrot et Anscombre (1977) pour le français moderne: un mais argumentatif et un mais réfutatif. Ce dernier s'oppose par ailleurs à un autre connecteur marquant la réfutation : ainz. La description du mais argumentatif du français moderne dans ses emplois canoniques est tout à fait valable pour le mais argumentatif de l'ancien français. Kleiber (1978) signale cependant, assez rapidement et avec certaines réserves, un emploi particulier de mais en ancien français, qu'il appelle «le mais de narration ». Comme il le signale lui-même: "L'étude de ce que nous avons sans doute abusivement appelé le mais de narration reste à faire» (p.276). C'est l'étude systématique de ce phénomène que nous entreprenons ici à partir d'une approche pragmatique et argumentative.

Nous avons abordé récemment le fonctionnement de mais et de ainz en ancien et en moyen français, tant d'un point de vue syntaxique que sémantique (cf. Rodríguez Somolinos 2000 et à paraître). Nous laisserons ici de côté mais réfutatif, pour analyser certains emplois particuliers que présente mais argumentatif en ancien français notamment le mais de narration signalé par Kleiber -. Cet emploi de mais est signalé rapidement par quelques linguistes et grammairiens, mais son étude systématique n'a pas été entreprise à ce jour. Notre étude s'appuie sur des textes littéraires en ancien français. En plus de nos propres dépouillements, nous avons eu recours à la Base de Français Médiéval, développée par le laboratoire d'Analyses de corpus linguistiques de l'ENS Lettres et Sciences Humaines de Lyon.

Notre hypothèse est tout d'abord que les emplois narratifs de mais ne peuvent être étudiés isolément. Il convient d'étudier de façon parallèle l'emploi de mais argumentatif dans les séquences dialogales, qui peut parfois être rapproché du mais présent dans les séquences narratives. Nous pensons d'autre part que, dans tous ces cas, mais a une valeur pleinement argumentative et qu'on peut le rattacher à la description générale de mais argumentatif que donnent O. Ducrot et J.-C. Anscombre pour le français moderne.

\section{Mais argumentatif}

6 La description sémantique de mais argumentatif est très connue. Voyons d'abord l'emploi standard de mais argumentatif en ancien français, avant d'aborder des emplois plus complexes.

7 Pour la théorie de l'argumentation dans la langue, le langage ordinaire n'a pas pour fonction principale de décrire le monde, de représenter la réalité. Un énoncé possède avant tout une orientation argumentative, le locuteur utilise cet énoncé pour argumenter dans un certain sens, pour amener son allocutaire à une certaine conclusion. La valeur argumentative d'un énoncé est constituée par les différents enchaînements auxquels il peut donner lieu dans le discours, par l'influence qu'il prétend exercer sur l'allocutaire.

8 Cette dimension argumentative est particulièrement présente dans le fonctionnement d'un connecteur comme mais. Dans une structure $p$ mais $q$, ce n'est pas le contenu informatif de $p$ et de $q$ qui doit être pris en compte. $P$ et $q$ sont des arguments, ils se présentent comme amenant l'allocutaire vers tel ou tel type de conclusion. Lorsqu'il énonce $p$ mais $q$, le locuteur adopte les deux attitudes suivantes :

1. Il présente $p$ comme un argument pour une conclusion $r$.

10 2. Il présente $q$ comme un argument pour la conclusion inverse non-r. 
11 Autrement dit, les propositions $p$ et $q$, visant des conclusions opposées, sont antiorientées argumentativement. Par ailleurs, $q$ est un argument plus fort en faveur de non-r que ne l'est $p$ en faveur de r. Par conséquent, la suite $p$ mais $q$ dans son ensemble est orientée vers non-r.

12 Cela ne signifie pas que le locuteur ne tienne pas compte de $p$. Faisant partie d'un mouvement concessif, $p$ est l'argument concédé. Le locuteur maintient $p$ comme un argument valable pour $r$, tout en lui opposant un argument plus fort pour la conclusion opposée non-r. Comme le signalent Ducrot/ Vogt (1979), le maintien de $p$ par le locuteur est graduel et peut se réaliser dans une plus ou moins grande mesure.

13 C'est là l'emploi standard de mais argumentatif, tout à fait courant en français moderne comme en ancien français. Nous verrons comme exemple un extrait du Voyage de Saint Brandan. Le saint et ses compagnons arrivent devant le mur qui protège le paradis :

\begin{tabular}{|l|l|l|}
\hline & $(1)$ & Tendent tut dreit vers la porte, \\
\hline & & Mais l'entree mult ert forte: \\
\hline & & Draguns i at qui la guardent ; \\
\hline & & Si cume fus trestut ardent. (Saint-Brandan, v.1704) \\
\hline
\end{tabular}

14 C'est là une structure $p$ mais $q$. Nous prendrons pour $p$ Tendent tut dreit vers la porte. Le narrateur présente $p$ comme un argument pour une conclusion $r$ «ils vont pouvoir franchir la porte ». La proposition q l'entree mult ert forte est orientée argumentativement vers la conclusion opposée non-r « ils ne peuvent pas la franchir ». La suite $p$ mais $q$ dans son ensemble est orientée vers non-r.

Notons que ce n'est pas le contenu informatif de $p$ et de $q$ qui est en cause ici. Mais établit une opposition, non pas entre les propositions $p$ et $q$, mais entre les conclusions argumentatives qu'elles visent. Le contexte discursif est essentiel pour déterminer l'orientation argumentative de $p$ et de $q$, il explicite parfois même la conclusion :

\begin{tabular}{|l|l|l|}
\hline & $(2)$ & « Vallez, fet il, gent et apert \\
\hline & & te voi molt, et de fier corage, \\
\hline & & mes trop par ies de juene aage: \\
\hline & & por ce me pans et sai de fi \\
\hline & & que, se je te vainc ou oci, \\
\hline & & que los ne pris n'i aquerroie, » (Cligès, v.4114) \\
\hline
\end{tabular}

16 Dans ce passage, $p$ gent et apert te voi molt, et de fier corage est orientée vers le combat, alors que $q$ trop par ies de juene aage est orientée en sens inverse, contre le combat. L'enchaînement discursif explicite la conclusion: le locuteur n'a pas l'intention de 
combattre, un combat dans ces conditions ne lui rapporterait pas d'honneur. La continuation discursive de $p$ mais $q$ enchaîne sur non-r.

17 Un cas particulier de mais argumentatif est ce qu'on a appelé le mais triangulaire, dans lequel $r$ est égal à non-q. En français moderne, ce type de structure se rencontre surtout lorsque mais relie deux adjectifs. L'exemple de G. Lakoff est devenu classique :

(3) Il est républicain, mais honnête.

18 Selon le schéma standard, la proposition $p$ Il est républicain est orientée vers une conclusion r « Il n'est pas honnête » qui équivaut en fait à non-q. Notons que dans ce type de structure, c'est $q$ qui permet de réinterpréter $p$ et de trouver la conclusion $r$. Comme le signale Schapira (1986: 507), cette structure sémantique présente un présupposé «Les républicains sont en général malhonnêtes»: «Pour que $p$ mais $q$ soit une phrase acceptable, il faut que A [le présupposé] soit une opinion que l'interlocuteur partage, une idée qu'il peut retrouver facilement parce qu'elle fait partie de son bagage de connaissances extralinguistiques, un préjugé, une idée reçue ou communément admise même si elle n'est pas partagée.» D'un point de vue argumentatif, ce présupposé correspond à ce que Ducrot appelle un topos, un principe général, présenté comme commun aux interlocuteurs, et qui sous-tend l'argumentation. Pour Schapira (1986: 507), ce mais introduit une exception, un homme qui, bien qu'il soit républicain, est honnête : " Mais déclare le sujet, en vertu de $\mathrm{p}$, différent des autres composants de la catégorie définie par A. C'est un mais d'exception ».

En ancien français, ce type de structure avec un mais triangulaire reliant deux adjectifs est possible, mais assez rare :

\begin{tabular}{|l|l|l|}
\hline & (4) & Et d'autre part une chapele \\
\hline & & Petite, mais ele est moult bele. (Lion, v.394, cité par Kleiber 1978) \\
\hline
\end{tabular}

Cette structure fait appel à un topos «les chapelles petites ne sont généralement pas belles ». Le mouvement argumentatif peut être paraphrasé comme suit : « La chapelle est petite, on pourrait en déduire qu'elle n'est pas belle, or ce n'est pas le cas, car j'affirme qu'elle est belle». Il s'agit bien d'un mais triangulaire, puisque $\mathrm{r}$ « la chapelle n'est pas belle » est égal à non-q.

21 Mais triangulaire est très fréquent en ancien français lorsque $q$ présente une négation syntaxique :

\begin{tabular}{|l|l|l|}
\hline & (5) & Dunc prent li pedre de se[s] meilurs serganz, \\
\hline & & Par multes terres fait querre sun amfant; \\
\hline & & Jusque an Alsis en vindrent dui errant: \\
\hline & & Iloc truverent danz Alexis sedant, \\
\hline
\end{tabular}




\begin{tabular}{|l|l|l|}
\hline & & Mais n'a(n) conurent sum vis ne sum semblant. (Alexis, v.115) \\
\hline & $(6)$ & «Jo desfiai Rollant le poigneor \\
\hline & & E Oliver e tuiz lur cumpaignun \\
\hline & & Carles l'oï e si nobilie baron. \\
\hline & & Venget m'en sui, mais n'i ad traïsun.» \\
\hline & & Respundent Francs : « A conseill en irums. » (Roland, v.3778) \\
\hline
\end{tabular}

On rencontre parfois en ancien français, surtout dans les textes du XIII ${ }^{\mathrm{e}}$ siècle, une suite $p$ mais q ainz $y$. Mais y est généralement triangulaire :

(7) Et chis li respondi qu'il n'en savoit mais nul, fors que on disoit qu'il avoit trois joules vaslés en le vile, qui estoient d'un lignage que on apeloit le lignage d'Angle, et estoient haut homme, mais il n'estoient mie rike, ains estoient povre ne n'estoient mie de grant pooir. (Clari, XXI)

La proposition $p$ et estoient haut homme «ils étaient de haute noblesse » est présentée comme un argument pour la conclusion $r$ «ils étaient riches». Cette conclusion vient s'opposer par ailleurs à $q$ « ils n'étaient pas riches ». D'un point de vue logique $r$ équivaut ici à non-q, il s'agit bien d'un mais triangulaire. Cette suite $p$ mais $q$ est suivie de ainz y ains estoient povre. Nous remarquons que $y$ est en fait coorientée avec $q$, elle vise le même type de conclusion. $Y$ constitue une paraphrase de $q:$ «ils étaient pauvres », «ils n'étaient pas riches ». Ainz réalise ici une réfutation qui vient renforcer le mouvement argumentatif mis en place par mais d'une façon qui est ressentie comme redondante.

\section{Un mais de narration en ancien français}

Les linguistes et grammairiens travaillant sur mais en ancien français ont signalé que la conjonction acquiert dans certains contextes, une valeur particulière. Il s'agit cependant de remarques brèves et isolées. La question n'a jamais été approfondie, par manque d'un cadre théorique permettant de décrire les différents effets de sens de mais argumentatif en contexte.

Antoine (1962 : 1119) signale que « mais sert à détacher un fait soudain et remarquable »:

\begin{tabular}{|l|l|l|}
\hline & (8) & Vait par les rues dunt il ja bien fu cointe, \\
\hline & & Altra pur altre, mais sun pedre i ancuntret, \\
\hline \hline & & Ansembl'ot lui grant masse de ses humes (Alexis, v.213). \\
\hline
\end{tabular}

Il faudrait traduire : «Il parcourt les rues, qui lui étaient jadis familières/ L'une après l'autre ; mais voici qu'il rencontre son père.. ». Faisant partie d'une séquence narrative, 
mais marque un tournant dans le récit. L'opposition qu'établit mais ne porte pas sur le contenu des propositions qu'il relie, mais sur la façon dont le texte se déroule. Les conclusions visées par $p$ et $q$ sont de type narratif. Le mouvement argumentatif imposé par mais peut se paraphraser ainsi: "Vous auriez tendance à croire que le récit va continuer dans le même sens, or vous vous trompez, car voici que survient un événement imprévu ».

Ménard (1976 : 271) signale également dans une remarque que mais «peut souligner un contraste au sens de et voici» :

\begin{tabular}{|l|l|l|}
\hline & (9) & As porz d'Espaigne en est passet Rollant \\
\hline & & Sur Veillantif, sun bon cheval curant. \\
\hline & & Portet ses armes, mult li sunt avenanz \\
\hline & & Mais sun espiet vait li bers palmeiant, \\
\hline & & Cuntre le ciel vait la mure turnant, \\
\hline & & Laciet en sum un gunfanun tut blanc; \\
\hline & Les renges d'or li batent josqu'as mains. \\
\hline & Cors ad mult gent, le vis cler e riant. (Roland, v.1155) \\
\hline
\end{tabular}

28 Ménard propose une traduction : «Il porte ses armes, elles lui vont très bien; Et voici que le baron brandit son épieu.» Nous avons affaire ici à un texte hétérogène mêlant étroitement des séquences descriptives et narratives, les propositions $p$ et $q$ reliées par mais ne sont pas en principe antiorientées. Mais ne semble pas au premier abord strictement nécessaire et le récit pourrait en faire l'économie. Le connecteur joue cependant un rôle au niveau du déroulement du récit. En instaurant artificiellement une opposition entre $p$ et $q$, mais permet d'insérer un segment narratif dans une séquence descriptive. Il sert en fait à introduire un rhème de façon emphatique, à mettre en relief un événement qui se détache de façon spéciale dans la description de Roland.

Le passage suivant de La vie de Saint Brandan est à rapprocher des précédents :

\begin{tabular}{|l|l|l|}
\hline & $(10)$ & Enz en la nef entré sunt tuit. \\
\hline & & Mais lur isle mult tost s'en fuit, \\
\hline & & E de dis liuues bien choisirent \\
\hline & & Le fou sur lui qu'il i firent. (Saint Brandan, v.464) \\
\hline
\end{tabular}

30 C'est l'épisode de l'île-baleine. Saint Brandan et ses compagnons ont abordé dans une île. Ils ont fait du feu pour préparer leur repas lorsque l'île, qui est en réalité une baleine, commence à trembler et à s'éloigner du bateau. Ils s'empressent de rejoindre leur bateau. 
Nous traduirions ici : «Ils réussirent tous à regagner le bateau. Mais voici que leur île s'éloigne à toute vitesse.» De $p$ le lecteur pourrait tirer une conclusion «le récit va continuer dans le même sens ». Mais présente ici $q$ - la fuite de l'île - comme quelque chose de surprenant par rapport à $p$. La conclusion non-r sera « un événement inattendu se produit ». La description sémantique de mais argumentatif donnée par Ducrot est donc applicable dans tous ces cas, même si mais fonctionne au niveau de la narration.

Voici encore un emploi similaire, signalé par Antoine (1962:1119) : «Au vers 141 de la Passion de Clermont se trouve un mais assez différent: il indique bien, sans doute, une certaine opposition entre ce qui précède et ce qui va suivre, mais il marque surtout un progrès dans l'action et reste proche encore du sens augmentatif du latin magis »:

\begin{tabular}{|l|l|l|}
\hline & $(11)$ & «- Eu soi aquel ! » zo dis Jesus \\
\hline & & Tuit li felun cadegrent jos ! \\
\hline & & Terce vez lor o demanded ; \\
\hline & & a totas treiz chedent envers ! \\
\hline & & Mais li felun tuit trassudad, \\
\hline & & vers nostre don son aproismad. (Passion, v.141). \\
\hline
\end{tabular}

32 Nous proposerions de traduire: "Mais voici que les félons, couverts de sueur, s'approchent de Notre Seigneur ». Mais ne serait pas ici strictement nécessaire. Par sa présence, il marque une transition dans le récit, il établit une frontière entre deux épisodes tout en constituant un lien qui permet au récit de progresser.

Cet emploi de mais introduisant un événement imprévu ou surprenant dans un récit est rare. Il se rencontre surtout dans des textes archaïques ou archaïsants. Plus généralement, mais fonctionne en ancien français comme un instrument de transition dans le récit. Cela a été remarqué par Kleiber (1978), qui prend comme point de départ la remarque de Moignet (1973: 334) selon laquelle «la conjonction peut indiquer simplement le passage à un autre genre de propos ». Kleiber $(1978: 275)$ établit ainsi, avec certaines réserves, l'existence d'un mais de narration : « Le rôle de mais dans ces exemples est d'introduire un élément de tension, une articulation logique, un pseudo-raisonnement argumentatif entre deux phrases qui, d'après leur contenu, n'exigent pas, de prime abord, une telle coordination. (...) Mais n'est pas indispensable pour assurer le passage. Sa présence a cependant pour effet d'obliger le lecteur de concevoir $q$ comme opposé argumentativement à $p$. »

Le rôle de mais comme opérateur de transition dans un récit peut être explicité par le texte même : 
(12)

Et saciés que, a celui point que Kahedins vint au pont et Kex li senescaus, estoit encore li rois Hoël au pont garder. Mais atant laisse ore li contes a parler de la tour et du castel, car bien avom ore devisé a cestui point tout ce ki i apartient, et retourne as deus cevaliers ki viennent au pont, pour conter conment il lour avint et conment Kahedins se combati grant piece encontre son pere. ( Tristan, 139, 41)

Le narrateur expose ici explicitement l'organisation de son récit. Il introduit un commentaire métalinguistique portant sur la narration. Mais vient ainsi souligner le passage d'un épisode à un autre. Encore une fois, mais ne serait pas strictement nécessaire. La formule atant laisse ore li contes a parler de - fréquente dans la prose du XIII ${ }^{\mathrm{e}}$ siècle - suffirait à articuler le récit. Mais $q$ s'oppose en fait à l'attente du lecteur, selon laquelle le récit va se poursuivre sans qu'il y ait un changement de thème.

Le plus souvent, la transition que mais rend possible n'est pas explicitée :

\begin{tabular}{|l|l|l|}
\hline (13) & Li quens Rollant gentement se cumbat, \\
\hline & & Mais le cors ad tressuët e mult chalt. \\
\hline & & En la teste ad e dulor e grant mal : \\
\hline & & Rumput est li temples, por ço que il cornat. \\
\hline & & Mais saveir volt se Charles i vendrat: \\
\hline & Trait l'olifan, fieblement le sunat. (Roland, v.2099) \\
\hline
\end{tabular}

Encore une fois, mais joue ici un rôle d'organisation textuelle. Les propositions $p$ et $q$ ne sont pas en principe antiorientées. C'est mais qui établit entre elles une opposition argumentative de type narratif.

Ce mais d'organisation textuelle est assez fréquent dans les textes :

\begin{tabular}{|l|l|l|l|}
\hline$(14)$ & $\begin{array}{l}\text {.. et fu si granz la meslee que poi i ot des rues ou il n'eüst granz estors d'espees et de lances et } \\
\text { d'arbalestes et de darz; et mult i ot genz navrez et morz. }\end{array}$ \\
\hline $\begin{array}{l}\text { Mais li Venisien ne porent mie l'estor endurer, si comencierent mult a perdre; (Villehardouin, } \\
89)\end{array}$ & $\begin{array}{l}\text { La ou je estoie a pié et mes chevaliers, aussi blecié comme il est devant dit, vint le roy atoute sa } \\
\text { bataille a grant noyse et a grant bruit de trompes et de nacaires, et se aresta sur un chemin levé. } \\
\text { Mes onques si bel armé ne vi, car il paroit de sur toute sa gent des les espaules en amon, un } \\
\text { heaume doré en son chief, une espee d'Alemaingne en sa main. (Joinville, 228) }\end{array}$ \\
\hline
\end{tabular}

Situé au début d'un paragraphe, mais joue en (14) un rôle de démarcation très évident. Il marque non seulement l'articulation du récit, mais aussi le découpage du texte dans sa matérialité. Dans tous ces emplois, mais se situe sur une frontière et en ce sens il constitue 
un signal démarcatif, mais il établit en même temps un lien, il efface la discontinuité en permettant au récit ou à la description de progresser. Comme le signale Maingueneau (1990: 66) en analysant ce qu'il appelle le mais romanesque du français moderne : «Le connecteur ne dissimule pas la ligne de rupture; il en change plutôt le statut. Mais distingue bien deux séquences pour les opposer (celle qui est concédée et celle qui est donnée comme plus forte), mais il s'agit d'une opposition argumentative, et non chronologique ou spatiale ».

Mais peut opérer également une transition dans des séquences descriptives :

\begin{tabular}{|l|l|l|}
\hline & (16) & Tuz ert entrins, sanz antaile, \\
\hline \hline & & Unc al faire n'out travaile, \\
\hline & & Mais les gemmes funt granz lüurs \\
\hline & & Dum purplantez esteit li murs. (Saint Brandan, v.1677-80) \\
\hline
\end{tabular}

Saint Brandan et ses compagnons arrivent en vue du Paradis, qui est entouré d'un mur blanc et lisse. Mais, dont la traduction est ici très difficile, structure la description, il lui permet de progresser. Le connecteur souligne de façon emphatique le passage à la description des gemmes.

Le narrateur peut utiliser mais pour abréger son récit :

\begin{tabular}{|l|l|l|}
\hline & $(17)$ & Enuiz serait a desraisnier \\
\hline & & et d'aconter trestoz les mes, \\
\hline & & qui sovent vindrent et espés, \\
\hline & & et a nomer vins et herbez, \\
\hline & & mais chascuns an i ot asez. (Enéas, v.832, cité par Antoine p. 1123) \\
\hline
\end{tabular}

Le narrateur fait ici une remarque portant sur le déroulement de son récit. Mais joue un rôle nettement narratif. $P$ est présenté comme un argument visant une conclusion $\mathrm{r}$ « Je ne vais pas raconter dans le détail le dîner d'Enéas et de Didon, je ne vais pas énumérer tous les plats qui ont été servis ». Q est orienté vers non-r « je vais quand même ajouter ce dernier détail ». Un mais tout à fait similaire se retrouve dans le Roman d'Enéas, v.9437.

Il est intéressant de rapprocher (17) du passage suivant, dans lequel le rôle narratif de mais est explicité par une formule présentant le verbe dire:

\begin{tabular}{|l|l|l|}
\hline & $(18)$ & car toute nuit dusqu'au demain \\
\hline & & dura cele tormente isi, \\
\hline
\end{tabular}




\begin{tabular}{|l|l|l|}
\hline & & ne nus d'ex d'ileuques n'isi, \\
\hline & & n'il n'i mangierent ne ne burent; \\
\hline \hline & & a la nue terre se jurent, \\
\hline n'il n'i orent nule rien plus; \\
\hline \hline & & je ne vous di rien du sorplus, \\
\hline 'il i orent autre delit, \\
\hline & & mais itant vous di que lor lit \\
\hline
\end{tabular}

45 Comme en (17), le narrateur utilise mais pour couper court à une énumération, pour abréger le récit. Le mouvement argumentatif est formulé ouvertement, je ne vous di rien du sorplus explicite $r$, alors que la formule itant vous di que énonce la conclusion non-r : «Je ne vais rien vous dire d'autre, je vous dirai cependant ceci ».

\section{4. mais à valeur énonciative en ancien français}

Dans les exemples que nous avons vus jusqu'ici, la suite $p$ mais $q$ apparaît dans des séquences narratives ou descriptives. Le connecteur renvoie à la narration, il réalise un commentaire sur le déroulement du récit ou de la description. Ces emplois narratifs de mais que nous venons d'analyser doivent être mis en parallèle avec certains emplois énonciatifs de mais dans des séquences dialogales. En effet, mais peut renvoyer à l'énonciation dans le dialogue dramatique ou dans le discours direct d'un personnage.

47 Nous avions vu en (12) que le rôle de mais comme opérateur de transition dans un récit peut être explicité par le texte même, à l'aide de formules du type atant laisse ore li contes a parler de. Un mais très similaire se rencontre dans le discours direct :

\begin{tabular}{|l|l|l|}
\hline & $(19)$ & "Sire compains, dist Amiles li ber, \\
\hline & & Il est bien drois par sainte charité \\
\hline & & Ques aillissiéz veoir et esgarder, \\
\hline & & Car sa moillier doit on bien honorer. \\
\hline & & Mais une chose voz voil dire et conter, \\
\hline & & Sire compains, que voz ne m'oubliéz. » (Amile, v.556) \\
\hline
\end{tabular}

Mais est ici tout à fait nécessaire pour éviter l'incohérence. La transition est lourdement explicitée de toute façon par la formule une chose voz voil dire et conter qui apparaît dans 
les textes avec des variantes: mais une chose voz di je par verté (Amile, v.1615), mais d'une chose vos vorroie proier (Charroi, v. 715, v.731). L'emploi du verbe dire ou d'un verbe de parole dans ces formules indique que la valeur de mais est clairement énonciative. Le locuteur utilise mais pour réaliser un commentaire sur sa propre énonciation. Le mouvement argumentatif en (19) est le suivant : « Vous auriez tendance à croire que c'est tout ce que j'ai à vous dire, or j'ajoute ceci, à savoir que vous ne devez pas m'oublier ».

Mais introduit très souvent une interrogation qui marque un changement de propos :

\begin{tabular}{|c|c|}
\hline \multirow[t]{2}{*}{$(20)$} & «Moi dist uns mires, qui iert de Besanson, \\
\hline & Qui me donna et herbes et puisons \\
\hline & Que en mon cors avoie grant frison, \\
\hline & Et que a fame n'eusce habitacion \\
\hline & Ne compaingnie tel com avoir doit on, \\
\hline & Ainz m'en tenisse trente jors a bandon. \\
\hline & Se nel faisoie, je sui sans garison. \\
\hline & Mais par la foi que devéz Deu del mont, \\
\hline & Por quoi haéz Amile le baron?» \\
\hline & "-Sire, dist elle, et noz le voz dirons» (Amile, v.1202) \\
\hline (21) & $\begin{array}{l}\text { "Signeur cevalier, encontrastes vous cha devant un cevalier ki portoit un escu vermeil a une } \\
\text { teste blance de lyon?" "Certes, fait Kex, nous l'encontrames voirement, orendroit k'il se } \\
\text { parti de nous. Mais savés vous ki il est?"- "Oill, ce dist li vallés. Ce est Lamorat de Gales, li } \\
\text { fiex Pellynor de Lystenois." (Tristan, 130) }\end{array}$ \\
\hline (22) & $\begin{array}{l}\text { "Vos en souvient il ore bien?"- "Certes, fait li rois, encore m'est il avis que ge la voie } \\
\text { l'assemblee, car onques puis en leu ou ge fusse ne vi autant fere d'armes a un chevalier comme } \\
\text { il fist celui jor. Mes por quoi l'avez vos dit?" (Mort Artu, 53, 1.35) }\end{array}$ \\
\hline
\end{tabular}

Le procédé est très courant dans les textes en ancien français. La suite $p$ mais $q$ apparait à l'intérieur d'un discours direct d'un personnage, et non pas en début de réplique. Mais $q$ réagit toujours a une proposition $p$ énoncée par le même locuteur et permet à celui-ci de réorienter son discours, de poser une question qui n'a pas de rapport avec ce qui précède. Mais est ici nécessaire, le locuteur ne pourrait pas poser la question sans qu'il y ait incohérence. Le mouvement argumentatif est le suivant: "De ce que je viens de dire, vous auriez tendance à croire que je vais continuer à parler dans le même sens, or vous vous trompez, car je préfère vous poser la question suivante ». Le connecteur mais sert à établir un lien avec le contexte antérieur, tout en justifiant la pertinence de la question. Notons que l'enchaînement porte sur l'énonciation, sur le fait de dire quelque chose et non sur le contenu de $p$. Le locuteur réalise un commentaire métalinguistique portant sur 
le déroulement de son propre discours. En ce sens, ces emplois sont à mettre en parallèle avec les emplois narratifs que nous avons vus ci-dessus.

Mais n'introduit pas toujours directement l'interrogation. Il peut être suivi par une formule avec le verbe dire à l'impératif :

\begin{tabular}{|l|l||l|l|}
\hline (23) & $\begin{array}{l}\text { «- En non Dieu, fait cil a la Cote Mautaillie, de ceste aventure sui je mout liés et mout } \\
\text { joians, car ausi voloie je cele part aler. Mais, pour Dieu, itant me dites : quel escu porte } \\
\text { mesire Lanselos, si que je le puisse connoistre se aventure m'amainne avoec lui ? » Et il li } \\
\text { devise. (Tristan, } 40)\end{array}$ \\
\hline \hline & $(24)$ & Ce dist li angres : « Ne te desesperer, \\
\hline & & Mais or me di, garde nel me celer, \\
\hline
\end{tabular}

52 Ce procédé permet d'introduire aussi une interrogation indirecte, comme on le voit en (24). La formule or me di, or me dites, itant me dites renvoie visiblement à l'énonciation et vient renforcer mais dans son rôle d'opérateur de transition. Elle annonce l'interrogation qui va suivre et en facilite ainsi l'apparition.

\section{Un mais causal en ancien français ?}

Melander (1916: 113) réunit dans le chapitre VI de sa thèse doctorale « Mais et ainz au sens causal » un certain nombre d'exemples de mais qu'il ressent comme différents. Pour les expliquer, il formule l'hypothèse que mais peut avoir en ancien français un "sens explicatif ». La thèse de Melander est une étude admirable à bien des égards, mais ce chapitre montre à quel point les connaissances linguistiques de l'époque ne permettaient pas d'analyser les expressions argumentatives. Dans certains cas, les exemples de mais que donne Melander correspondent à l'emploi standard de mais argumentatif. Dans d'autres, il s'agit du mais de narration que nous avons décrit ci-dessus. D'autres, finalement, correspondent à des emplois dialogaux dans lesquels mais présente une valeur énonciative. En aucun cas, à notre avis, on ne peut parler d'un sens causal de mais. Il est vrai que Melander (1916 : 115) lui-même émet quelques réserves sur son analyse : «Dans bien des cas, le sens causal n'est guère perceptible. Il s'agit de nuances de sens extrêmement subtiles. »

Voici un mais que Melander présente comme causal :

\begin{tabular}{|l|l|l|}
\hline & $(25)$ & Quant an la cambra furent tut sul remés, \\
\hline & & Danz Alexis la prist ad apeler ; \\
\hline & & La mortel vithe li prist mult a blasmer, \\
\hline & & De la celeste li mostret veritét ; \\
\hline
\end{tabular}


Mais lui est tart quet il s'en seit turnét. (Alexis, v.65) pourquoi Alexis « prist a blasmer la mortel vide ». Le ms. A porte aussi : " car lui ert tart. » Cette dernière remarque explique ce qui a induit Melander en erreur. Car apparaît parfois dans les manuscrits comme une variante de mais, ce qui ne veut dire aucunement que leur fonctionnement sémantique soit identique. En tout cas, si Alexis tient des discours édifiants à sa jeune femme, ce n'est pas parce qu'il est pressé de partir. A notre avis, mais marque ici une transition dans le récit, il permet au narrateur de couper court au discours rapporté d'Alexis et de passer à autre chose. Il joue un rôle d'organisateur textuel et il est à rapprocher des exemples que nous avons vus ci-dessus. Le mouvement argumentatif serait le suivant: "On aurait tendance à croire que le narrateur va continuer à rapporter les propos d'Alexis, or ce n'est pas le cas car il préfère passer à autre chose ».

Melander donne également comme causal le passage du Roman d'Enéas (v.9437) que nous avons analysé ci-dessus en (17) dans lequel un mais narratif sert à couper court à une énumération. Il donne par ailleurs des exemples de mais dans des séquences dialogales qui ont, d'après lui, un sens causal :

\begin{tabular}{|l|l|l|}
\hline & $(26)$ & "Si m'ait Dex, mout me mervel \\
\hline & & Que mes niés ma vergonde ait quise \\
\hline & & Mais servi m'a d'estrange guise.» (Tristan 630, cité par Melander p. 115) \\
\hline
\end{tabular}

La suite $p$ mais $q$ fait partie du discours direct d'un personnage. L'opposition qu'établit mais entre $p$ et $q$ est de type énonciatif. Le locuteur fait au moyen de mais une remarque métalinguistique portant sur sa propre énonciation. $P$ est orienté vers une conclusion «je dis ceci à contrecoeur ", alors que $q$ vise une conclusion " je dois quand même le dire ».

Moignet (1973: 334) signale également que mais «peut prendre valeur explicative, introduire une justification » :

\begin{tabular}{|l|l|l|}
\hline & (27) & Tervagan, par melancolie \\
\hline & & Vous ai hui dit mainte folie; \\
\hline \hline & & Mais g'iere plus ivres que soupe. (Saint Nicolas, 165) \\
\hline
\end{tabular}

Il s'agit en fait d'un emploi tout à fait habituel de mais argumentatif, comme le signale d'ailleurs Kleiber (1978: 274). Il est vrai que $q$ donne les causes de $p:$ si le roi a dit des sottises à Tervagan, c'est parce qu'il était ivre. On ne peut cependant pas classer les emplois de mais en fonction du contenu informatif de $p$ et de $q$. Cela produirait autant d'emplois de mais que d'occurrences du connecteur. Nous proposons l'analyse suivante $: p$ est orienté vers une conclusion «j'ai fait exprès de vous dire des sottises ", alors que la 
justification fournie par $q$ «j'étais ivre» constitue en fait une excuse. L'ensemble de la suite $p$ mais $q$ réalise un acte d'excuse.

Nous croyons donc que ce mais causal ou de justification n'existe pas. Il correspond à différents effets de sens que peut prendre mais argumentatif en contexte.

\section{Mais opérateur de transition}

61 Le mais argumentatif de l'ancien français est similaire dans son emploi standard à celui du français moderne et, en ce sens, il peut être décrit conformément à la description sémantique qu'en donne $\mathrm{O}$. Ducrot. Mais argumentatif présente cependant dans ces deux langues des emplois particuliers. Comme nous l'avons vu ci-dessus, on peut bien parler d'un mais de narration pour l'ancien français. Il faut rapprocher cet emploi d'un mais énonciatif à l'œuvre dans les séquences dialogales. Il conviendrait donc de parler, d'un point de vue plus général, d'un mais opérateur de transition qui renvoie soit à la narration, soit à l'énonciation. Dans les deux cas, mais concerne l'organisation interne du texte ou du discours, son agencement, la façon dont il se déroule. Il s'agit cependant toujours d'un mais argumentatif pour lequel le schéma descriptif de Ducrot est tout à fait valable.

On ne peut pas établir de parallèle entre les emplois narratifs de l'ancien français et ceux que l'on rencontre dans les séquences narratives ou descriptives en français moderne. D'une part l'ancien français présente des emplois de mais qui lui sont propres. C'est le cas par exemple du mais servant à introduire un événement imprévu, comme nous l'avons vu en (8) ou (9). En français moderne, par ailleurs, les mais narratifs - ou descriptifs peuvent faire partie d'un phénomène très complexe, comme le montre bien l'analyse de Maingueneau (1990), à la suite de celles de Ducrot (1980) dans Analyses pragmatiques. Il s'agit très souvent d'un mais qui renvoie à une pensée représentée, il permet d'appréhender les événements racontés ou la scène décrite à travers le point de vue d'un personnage. Le mouvement argumentatif imposé par mais exige d'abord la réinterprétation de $p$ et permet d'établir un discours indirect libre. Ce rôle de mais comme organisateur textuel et comme créateur d'un sujet de conscience - tel qu'il a été étudié par Rabatel (1999) - nous semble relativement moderne, il est attesté notamment dans le récit classique du XIX ${ }^{e}$ siècle, il est inexistant en tout cas au moyen âge.

63 En ce qui concerne le mais dialogal énonciatif de l'ancien français, nous avons signalé qu'il apparait généralement dans une suite $p$ mais $q$ située à l'intérieur d'un discours direct. Le locuteur, ayant énoncé $p$, réoriente son discours à l'aide de mais q. Cela lui permet le plus souvent d'introduire une interrogation ou de changer de propos sans qu'il y ait incohérence.

64 En ancien français le mais dialogal qui apparait en début de réplique, assez rare d'ailleurs, correspond à un mais réfutatif qui vient réfuter ou rectifier les propos de l'allocutaire. Dans le dialogue suivant, Pincedé et Rasoir se disputent des besants :

\begin{tabular}{|l|l|l|}
\hline & $(28)$ & Rasoirs : Lais les! \\
\hline & & Pincedés: Mais tu, oste tes mains. \\
\hline & & Que je ne te crieve les iex! (Saint Nicolas, v.1165) \\
\hline
\end{tabular}



réplique. Il enchaîne toujours sur une proposition $p$ proférée par le même locuteur que mais $q$. En français moderne, par contre, mais argumentatif peut apparaître en début de réplique. Ducrot et al (1980) présente une classification des différents emplois de mais argumentatif dans le dialogue, à partir de l'analyse de Occupe-toi d'Amélie de Feydeau. Cette étude démontre que mais peut enchaîner en français moderne sur du non-verbal. Il peut marquer l'opposition du locuteur à un comportement de l'allocutaire ou à une situation. Cet emploi est inexistant en ancien français. Il apparaîtra en moyen français, il est visible notamment dans les farces de la fin du moyen âge.

\section{BIBLIOGRAPHIE}

\section{Etudes}

ADAM, J.-M. (1990), Eléments de linguistique textuelle, Liège : Mardaga, 191-211.

ANSCOMBRE, J.CL. et DUCROT, O. (1977), « Deux mais en français ? », Lingua 43, 23-40.

ANTOINE, G. (1962), « L'opposition mais/ains (ainçois) », dans La coordination en français, Paris, d'Artrey, vol. 2, 1114-1157.

DUCROT, O. (1978), « Deux mais », Cahiers de linguistique 8, 109-120.

DUCROT, O. et VOGT, C.A. (1979), « De magis à mais : une hypothèse sémantique », Revue de linguistique romane $52,317-341$.

DUCROT, O. (1980), « Analyses pragmatiques », Communications 32, 11-60.

DUCROT, O et al. (1980), « Mais occupe-toi d'Amélie », Les mots du discours, Paris : Ed. de Minuit, 93-130.

KLEIBER, G. (1978), « Sur l'emploi adversatif de mais et de ainz (ainçois) en ancien français », Tra Li Li 16, 1, 271-292.

MAINGUENEAU, D. (1990), Pragmatique pour le discours littéraire, Paris : Bordas, 63-66.

MELANDER, J. (1916), Etude sur magis et les expressions adversatives dans les langues romanes, Uppsala : Imprimerie Almqvist \& Wiksell.

MENARD, P. (1976), Syntaxe de l'ancien français, Bordeaux : Sobodi.

MOIGNET, G. (1973), Grammaire de l'ancien français, Paris : Klincksieck.

PONCHON, T. (1990), « Observations sur le connecteur mais en français médiéval », L'information grammaticale $46,47-51$.

RABATEL, A. (1999), « Mais dans les énoncés narratifs. Un embrayeur du point de vue et un organisateur textuel », Le français moderne 67, 1, 49-60. 
RODRIGUEZ SOMOLINOS, A. (2000), « Mais, ains, ainçois en moyen français : syntaxe et sémantique ", Le moyen français 46-47, 449-465.

RODRIGUEZ SOMOLINOS, A. (à paraître), « Ainz et mais en ancien français ». Romania. SCHAPIRA, C. (1985) « Mais entre deux adjectifs », Linguisticae Investigationes IX, 1, 97-107.

Textes de référence

Ami et Amile, éd. P. F. Dembowski, Paris : Champion, (CFMA 97) 1969.

L'Atre Périlleux, roman de la table ronde, éd. Brian Woledge, Paris : Champion, 1936.

BENEDEIT, Le voyage de Saint-Brandan, éd. I. Short et B. Merrilees. Paris : 10/18, 1984.

La chanson de Roland, éd. G. Moignet. Paris : Bordas, 1969.

CHRETIEN DE TROYES, Cligès, éd. A. Micha, Paris : Champion, (CFMA 84) 1957.

CHRETIEN DE TROYES, Yvain, le chevalier au lion, éd. Mario Roques, Paris : Champion, (CFMA 89) 1960.

Enéas, éd. J. J. Salverda de Grave, 2 vol., Paris : Champion, (CFMA, 44 et 62) 1973 et 1983.

JEAN BODEL, Le jeu de Saint Nicolas, éd. A. Jeanroy, Paris : Champion, (CFMA 43) 1982.

JEAN DE JOINVILLE, La Vie de Saint Louis, éd. J. Monfrin, Paris : Garnier Flammarion, 1966.

La Mort le Roi Artu, éd. Jean Frappier, Genève : Droz, [TLF] 1954.

ROBERT DE CLARI, La Conqueste de Constantinople, éd. Philippe Lauer, Paris : Champion, [CFMA, 40] 1974.

Tristan en prose (tome 1), éd. Philippe Ménard, Genève : Droz, [TLF] 1987.

La vie de Saint Alexis, éd. Ch. Storey. Genève-Paris: Droz Minard, [TLF] 1968.

VILLEHARDOUIN, G. de La conquête de Constantinople, éd. E. Faral. Paris : Les Belles Lettres, 1938, 2 vols. Col. « Les classiques de l'histoire de France au moyen-âge », n 18-19.

\section{AUTEUR}

\section{AMALIA RODRÍGUEZ SOMOLINOS}

Universidad Complutense de Madrid 